\title{
CSMA Self-Adaptation based on Interference Differentiation
}

\author{
Jing Zhu' ${ }^{1}$, Xingang $\mathrm{Guo}^{1}$, Sumit Roy ${ }^{2}$, and Konstantina Papagiannaki ${ }^{3}$ \\ 1: Communication Technology Lab, Intel Corporation, Hillsboro, OR 97124, USA \\ 2: University of Washington, Seattle, WA 98195, USA \\ 3: Intel Research Pittsburgh, 4720 Forbes Avenue, Suite 410, Pittsburgh, PA 15213 \\ \{jing.z.zhu, xingang.guo\}@intel.com, sroy@ee.washington.edu, and dina.papagiannaki@intel.com
}

\begin{abstract}
- this paper addresses the design challenge of interference mitigation in the emerging high density (HD) wireless LAN. It is proposed to differentiate interference according to their energy and timing relative to desired signal, and measure packet error rate (PER) locally at transmitter for each type of interference. Then, self-adaptation algorithms are designed to adjust a) clear channel assessment (CCA) threshold, aka physical carrier sensing threshold, to leverage spatial reuse for achieving higher aggregate throughput, and b) transmit power (TP) to compensate location difference among links, and prevent individual links from starving. Compared to an end-to-end (E2E) feedback loop, ours has negligible complexity and zero over-the-air overhead. Extensive OPNET simulations are used to compare the performance of our solutions against the legacy and the ideal. Key words: CSMA, adaptation, and CCA
\end{abstract}

\section{INTRODUCTION}

Over the past few years, there has been tremendous growth in the number of mobile computing devices exemplified by laptops, PDAs and other handheld units. In May 2005, more laptops than desktops were sold in the United States - a milestone in PC sales history [1]. The availability of mature IEEE 802.11 [2] wireless LAN technology in portable units provides a clear thrust behind this growth. The convenience and flexibility of wireless networking has caused 802.11 to emerge from the individual home (the market for which it was initially targeted) to large-scale deployments in environments covering medium to large enterprises, apartment complexes and housing developments, and public area hot-spots. Such networks comprise a large number (10s-100s) of Access Points (AP) that are separated by only a few meters on average [3], forming a multi-cell network serving a large number of clients (100s -- 1000s).

In such high-density (HD) networks, coverage is less of a design concern because of the ubiquitous deployment of APs. Rather, effective interference mitigation becomes the primary means to improve total network throughput. Multiple works [4]-[12] have established the role of CSMA adaptation in interference mitigation, through CCA (clear channel assessment) threshold tuning, TPC (transmit power control), or CW (contention window) adjustment. However, practical solutions for run-time application in actual networks are yet to be achieved, while there is no systematic approach that integrates various adaptation solutions.

The first step to improved CSMA adaptation is the ability to differentiate interference and disambiguate (even approximately) the various causes of packet loss. Fig. 1 shows three scenarios with various timing relations between the desired and the interfering signals, which we denote as type-1, type-2, and type-3 interference, respectively:

i) Type-1 interference results from an (weak) interfering signal unnecessarily deferring the transmission of the desired signal, i.e., the exposed terminal problem;

ii) Type-2 interference arises when multiple transmitters initiate transmission simultaneously in the same slot, termed as collision;

iii) Type-3 interference captures the well known hidden terminal problem, and the interferer cannot sense the ongoing transmission and initiates a transmission that will corrupt the desired signal.

RTS/CTS exchange has been proposed in [2] to address the hidden terminal problem. However, it adds additional and non-negligible overhead particularly for transmissions with small payload size.

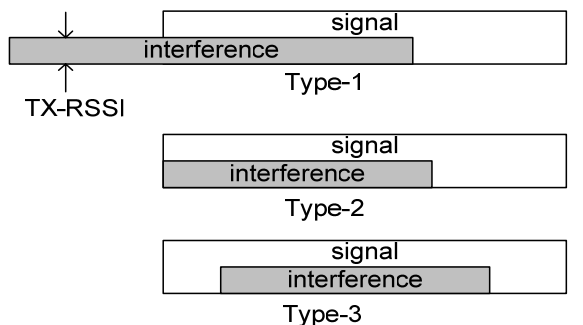

Fig.1 Interference differentiation

Differentiating the type of interference allows for the selection of an appropriate counter measure. For type-1 interference, if the reference transmission could be successfully decoded in the presence of the interfering signal, then an appropriate solution is to adjust the CCA threshold of the transmitter so as to avoid unnecessary deference. For type- 2 interference, an appropriate solution would be the tuning of the CW (contention window) size, so that the probability that multiple transmitters will transmit at the same time is minimized. Lastly, for type-3 interference due to hidden terminals, TPC would provide a potential solution, since it will raise the power level of the desired signal to a level that either cannot be corrupted by the interfering signal, or can reach the hidden interferer and force its deference.

In this paper, we propose techniques to differentiate the three interference cases (shown in Fig.1) based on the energy and timing of the two signals and measure local PER at transmitter side for each type of interference individually. We then design self-adaptation mechanisms for CCA threshold and transmit power. The rest of the paper is organized as follows. We present our techniques for interference differentiation in Section II, and for TPC and CCA self-adaptation in Section 
III. In Section IV, we show simulation results along with detailed analysis and discussion. Finally, we review related work in Section V, and conclude the paper in Section VI.

\section{INTERFERENCE DIFFERENTIATION}

We first introduce a new metric - TX-RSSI (transmit received signal strength indicator. As shown in Fig.1, it measures the over-the-air energy observed by a transmitter prior to a transmission. We then define CCAmin to measure the noise floor, which also indicates he minimum CCA threshold, i.e., a signal with energy level less than CCAmin will be treated as noise and never trigger "CCA busy". In other word, CCAmin provides the lower-bound to the adaptation of CCA threshold. Nevertheless, CCAmin itself could adapt to environment as discussed further in the next section.

For convenience, we denote the binary variable $E=\{T X$ $R S S I \geq C C A m i n\}$, which takes value $E=1(0)$ if type- 1 interference is detected (not detected). We then measure the number of transmissions and failures when type- 1 interference is detected (not detected):

$t_{1}$ : number of total transmissions with $E=1$

$f_{1}$ : number of failures with $E=1$

$t_{2}$ : number of transmissions with $E=0$

$f_{2}$ : number of failures with $E=0$

The goal is to measure PER for type-1, type-2, and type-3 interference individually, defined as

$p_{1}$ : the probability of packet loss caused by type- 1 interference, given $E=1$

$p_{2}$ : the probability of experiencing type-2 interference (collisions), given $E=0$

$p_{3}$ : the probability of packet loss caused by type-3 interference, given $E=0$

We also define the following notations to facilitate analysis:

$p$ : the probability of packet loss, given $E=1$

$\overline{p_{1}}$ : the probability of packet loss caused by non type-1 interference, given $E=1$

Further, we will use \langle\rangle around any quantity to denote its estimate based on observed data. Assuming packet losses caused by different types of interference are independent ${ }^{1}$, we have

$$
1-p=\left(1-p_{1}\right)\left(1-\overline{p_{1}}\right)
$$

where we estimate $p$ and $p_{1}$ via

$$
\langle p\rangle=\frac{f_{1}}{t_{1}} \text { and }\left\langle\overline{p_{1}}\right\rangle=\frac{f_{2}}{t_{2}} .\left(t_{1}>0, t_{2}>0\right)
$$

Wherein we measure the probability of packet loss caused by non type- 1 interference at $E=0$, with the assumption that it is

\footnotetext{
${ }^{1}$ Certainly, this cannot be strictly true, since given the $E=1$ (type-1 interference exists), the contribution of type-2 or type-3 interference to packet loss depends on the amount of type- 1 interference present.
}

independent of whether type- 1 is present or not. Now, we can obtain $\left\langle p_{1}\right\rangle$ by combining Eq.(1) and (2) as

$$
\left\langle p_{1}\right\rangle=1-\frac{1-\langle p\rangle}{1-\left\langle\overline{\left.p_{1}\right\rangle}\right.}=1-\frac{1-\frac{f_{1}}{t_{1}}}{1-\frac{f_{2}}{t_{2}}}, \quad\left(t_{1}>0, t_{2}>0\right)
$$

providing an estimate for PER due to type-1 interference.

Next, we will derive an estimator for $p_{2}$.

Based on the fact that type-2 interference (collision) starts at the same time as the signal, we propose a simple collision detection mechanism - each node delays its transmission by half slot $^{2}$ with probability q (say 0.5 ). This allows us to estimate $p_{2}$ without any significant impact on network throughput. For nodes that delay transmission, the first halfslot is used to measure the over-the-air energy for collision detection. We perform the following measurements:

$n$ : the number of delayed transmissions at any node;

$m$ : the number of delayed transmissions at any node whose energy level measured in the first half slot is $\geq$ CCAth (the current CCA threshold).

Assume that $N$ devices contend for the channel along with the reference node. If we denote the transmission probability for device $i$ as $\tau_{i}$, the collision probability for the reference device is then given by:

$$
p_{2}=1-\prod_{i=1}^{N}\left(1-\tau_{i}\right) \approx \sum_{i=1}^{N} \tau_{i}
$$

for $\tau_{i}<<1$. With the proposed delay, a transmission will be detected with probability $\tau_{i}(1-q)$.

Hence, the collision probability observed $(\mathrm{m} / \mathrm{n}$ ) equals

$$
\frac{m}{n}=\left\langle 1-\prod_{i=1}^{N}\left(1-\tau_{i}(1-q)\right\rangle \approx\left\langle(1-q) \sum_{i=1}^{N} \tau_{i}\right\rangle\right.
$$

We then get $\left\langle p_{2}\right\rangle$ by combining Eq.(4) and (5) as

$$
\left\langle p_{2}\right\rangle=\left(\frac{m}{n}\right)\left(\frac{1}{1-q}\right), \quad(n>0)
$$

Finally, $t_{2}-t_{2}\left\langle p_{2}\right\rangle$ measures the number of transmissions with neither type- 1 nor type- 2 interference in the measured interval and $f_{2}-t_{2}\left\langle p_{2}\right\rangle$ gives the number of failures due to the type-3 interference assuming that all collisions result in failures. Hence, we get the measured PER due to type-3 interference

$$
\left\langle p_{3}\right\rangle=\frac{f_{2}-t_{2}\left\langle p_{2}\right\rangle}{t_{2}\left(1-\left\langle p_{2}\right\rangle\right)}
$$

In summary, Eq.(3), (6), and (7) give the PER estimates to type-1, type-2, and type-3 interference, respectively.

\footnotetext{
${ }^{2}$ A slot is $9 \mu \mathrm{s}$ for $.11 \mathrm{a}$ or $.11 \mathrm{~g}$, and $20 \mu \mathrm{s}$ for $.11 \mathrm{~b}$. Notice even if a collision is detected, the transmission will still proceed.
} 


\section{SELF-AdAPTATION ALGORITHMS}

Our goal is to use CCA adaptation and TPC for type- 1 and type-3 interference, respectively. And we leave how to mitigate type-2 interference (collision) to the future.

We define a PER threshold, denoted as $P E R_{t h}{ }^{(C C A)}$, such that

$$
\begin{aligned}
& \text { If }\left\langle p_{1}\right\rangle<P E R_{t h}{ }^{(C C A)} \\
& \text { Then CCAth }=\min (C C A m a x, C C A t h+1 \mathrm{~dB}) \\
& \text { Else CCAth }=\max (\text { CCAmin, CCAth }-1 \mathrm{~dB})
\end{aligned}
$$

where CCAth, CCAmax, and CCAmin indicates the current, the maximum, and the minimum CCA thresholds allowed, respectively. As discussed in the previous section, CCA threshold of a transmitter only impacts type- 1 interference that its transmission will receive, and CCAmin is the threshold to detect type-1 interference. It is therefore desirable to adjust CCAmin to maintain a reasonable type- 1 interference detection ratio, measured by $t_{1} /\left(t_{1}+t_{2}\right)$. Detecting type- 1 interference does not necessarily lead to deference, which is governed by the actual CCA threshold (CCAth). Let's set the target range to $(10 \%, 90 \%)$, and adjust CCAmin as following:

$$
\begin{aligned}
& \text { If }\left(\frac{t_{1}}{t_{1}+t_{2}} \geq 90 \%\right), \\
& \quad \text { CCAmin }=\min (\text { CCAmax, CCAmin }+1 \mathrm{~dB}) \\
& \text { If }\left(\frac{t_{1}}{t_{1}+t_{2}} \leq 10 \%\right) \\
& \text { CCAmin }=\max (\text { CCAdef, CCAmin }-1 \mathrm{~dB})
\end{aligned}
$$

CCAdef is the default minimum CCA threshold, which is usually fixed, and defined by thermal noise. CCAmax is set to make sure that stations within a cell should hear each other ${ }^{3}$. We have:

\section{CCAmax $\geq$ CCAth $\geq$ CCAmin $\geq$ CCAdef.}

Generally, the lower the CCA threshold, the less aggressive the device is in attempting channel access, leading to less transmission opportunity. To prevent a device from starving, i.e. attempting too few transmissions, we choose $P E R_{t h}{ }^{(C C A)}$ as

$$
P E R_{t h}{ }^{(C C A)}=\left\{\begin{array}{cc}
0.01, & M \geq 20 \mathrm{pkt} / \mathrm{sec} \\
0.5, & 20 \mathrm{pkt} / \mathrm{sec}>M \geq 10 \mathrm{pkt} / \mathrm{sec} \\
1, & M<10 \mathrm{pkt} / \mathrm{sec}
\end{array}\right.
$$

where $M$ denotes the measured transmission opportunities (i.e. packet transmission rate). If $M$ exceeds what we consider a normal transmission rate, we will set $P E R_{t h}{ }^{(C C A)}$ equal to 0.01 . In that way spatial reuse will be leveraged carefully by increasing the CCA threshold only if $\left\langle p_{1}\right\rangle$ is insignificantly small, i.e. $<1 \%$. Otherwise, the adaptation will enter a more aggressive mode. If $M<10 \mathrm{pkt} / \mathrm{sec}$, the CCA threshold will increase regardless of the reliability of the link; if $10 \mathrm{pkt} / \mathrm{sec} \leq M<20 \mathrm{pkt} / \mathrm{sec}^{4}$, the transmission opportunity is still below the normal and the CCA threshold

\footnotetext{
${ }^{3}$ If the RSSI at cell edge with the minimum transmit power is $x(\mathrm{dBm})$, CCA threshold should be no higher than $x-3 \gamma(\mathrm{dBm})$, where $\gamma$ is path loss exponent. Because the maximum distance between two STAs in a cell is twice large as the cell radius. As an example example, CCAmax $\leq_{x-9}(\mathrm{dBm})$ if $\gamma=3$

${ }^{4}$ Assuming packet size 2000 bytes, 20pkt/sec means 320kbps, and 10pkt/sec means $160 \mathrm{kbps}$.
}

will increase only when $\left\langle p_{1}\right\rangle \geq 0.5$. Different values can be configured in (9), but they will not change the basic behavior of the algorithm. Due to space and time limitation, we leave the work of optimizing these parameters to the future. Here, we assume that they are fixed as in (9).

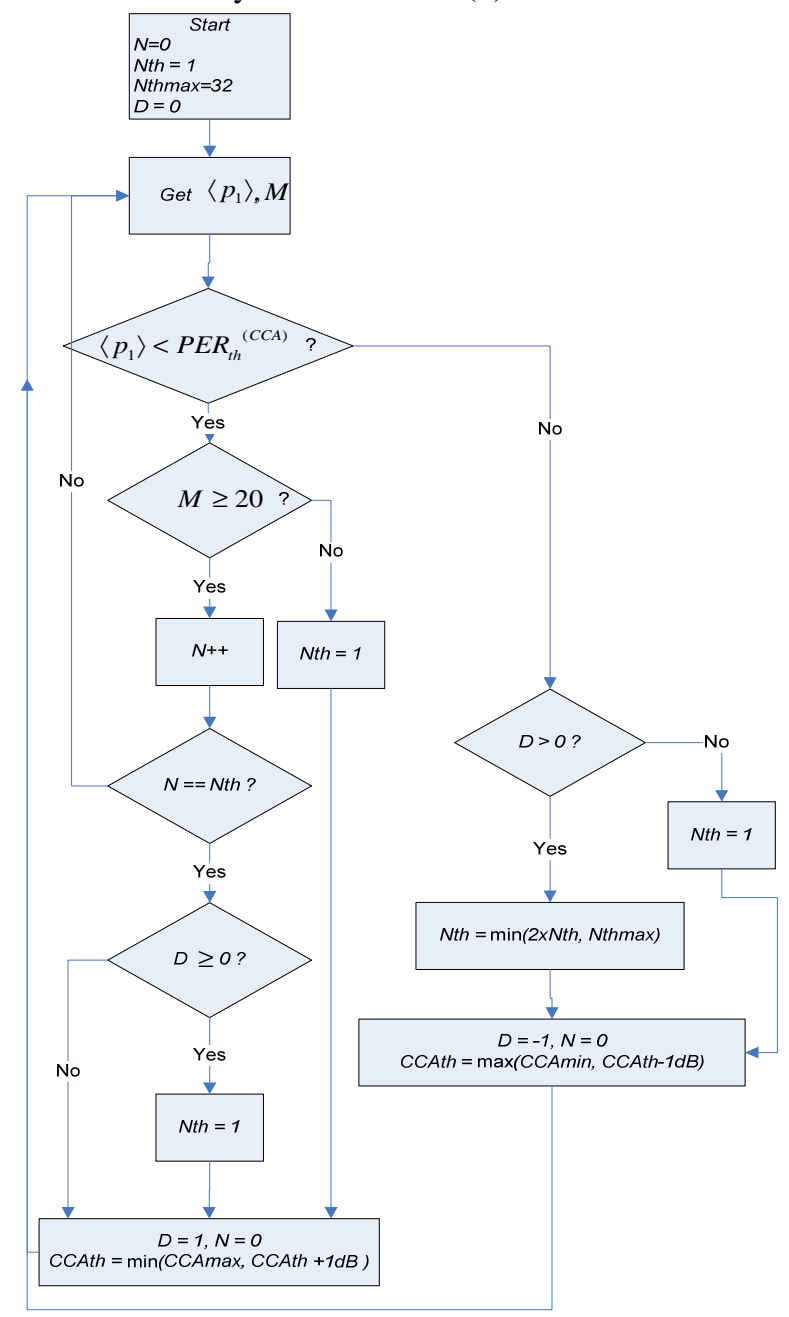

Fig.2 Flow chart of CCA adaptation algorithm

Oscillation, defined as "one increase followed by one decrease", indicates unnecessary adjustment (increase or decrease) of the parameter. To detect oscillation, we introduce another parameter, called adaptation indicator, denoted as $D$. It will record the direction of the last move: -1 for "decrease" and +1 for "increase". Oscillation is detected whenever the algorithm decides to reduce its CCA threshold while the last move was "increase". Another counter, denoted as $N$, is used to record the number of consecutive

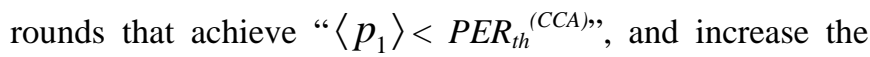
CCA threshold only when $N$ exceeds $N$ th. Nth is itself a variable between 1 and 32; the larger the value of $N$ th, the more conservative the increase of the CCA threshold. Whenever an oscillation is detected, Nth will double; otherwise, it will be set back to the minimum (=1). Successive oscillations will result in exponential increase of $N$ th, and the number of oscillations will be quickly minimized. Notice that the above anti-oscillation mechanism is enabled only when transmission opportunity is in the 
normal range, i.e. $M \geq 20$. Fig. 2 shows the flow chart of the proposed CCA adaptation algorithm.

TPC is very similar to the above with the main difference that it is driven by $\left\langle p_{3}\right\rangle$, i.e.

$$
\text { If } \begin{aligned}
\left\langle p_{3}\right\rangle<P E R_{t h}{ }^{(T P)} \text { Then } T P & =\max (T P \min , T P-0.5 \mathrm{~dB}) \\
\text { Else } T P & =\min (T P \max , T P+0.5 \mathrm{~dB})
\end{aligned}
$$

with the following notations:

TPmin: the minimum transmit power

TPmax: the maximum transmit power

$T P$ : the current transmit power

$P E R_{t h}{ }^{(T P)}$ : the PER threshold for TPC

TP increase will cause higher interference level to other devices, and therefore it is triggered only as necessary. Hereby, we adopt a small adaptation step, $0.5 \mathrm{~dB}$, and a single, high PER threshold $\left(P E R_{t h}{ }^{(T P)}=0.5\right)$. We will keep TP low as long as the PER due to hidden terminal is not too high $(<0.5)$. Again the goal is to prevent link starvation. Additionally, the same method is used to prevent oscillation.

Both TPC and CCA adaptation we proposed in this section are conducted by a WLAN device itself periodically. AP might have multiple links at the same time so that the adaptation should be conducted per either every STA (i.e. TP and CCA threshold for transmitting to different STAs are separately maintained) or the worst STA (i.e., the maximum PER estimate of all connected STAs).

In addition, we notice that most of today's WLAN radios allow transmit power only in a few fixed levels. For example, Cisco $1130 \mathrm{~A} / \mathrm{B} / \mathrm{G}$ AP can only take the value in the set $\{-1$, $2,5,8,11,14$, and 17$\}(\mathrm{dBm})$ for.11g mode. We believe that such limitation is not caused by hardware capability, but rather the lack of practical and effective TPC solutions tailored for WLAN system.

\section{SimUlation RESUlTS AND DisCUSSIONS}

We use OPNET v11.0 for the following simulation. Transmission rate is fixed since OPNET does not support rate adaptation. Different rates are nonetheless investigated in order to make sure that the proposed solution can bring benefits independently of the rate setting.

A. Two-Cell - the joint power of TPC and CCA adaptation

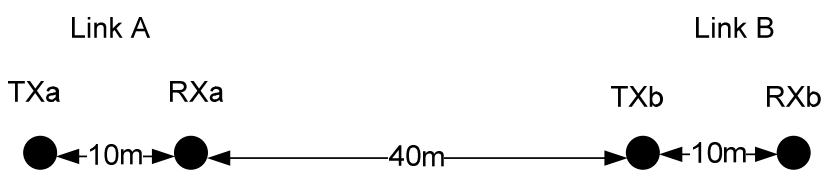

Fig.3 Two link competing scenario $(\gamma=3)$

First of all, we use a simple two-cell scenario to demonstrate how TPC and CCA adaptation algorithms work together to improve throughput and fairness performance. Fig. 3 shows the network topology. The black arrow indicates the direction of data transmission. In our simulation, we use saturated UDP traffic with packet size 2000 bytes, and fixed 802.11g rate 54 Mbps. We use $\gamma$ to denote path loss exponent, and configure TPmin $=14 \mathrm{dBm}$, TPmax $=20 \mathrm{dBm}$, CCAdef $=-86 \mathrm{dBm}$, and
CCAmax $=-66 \mathrm{dBm}$. The RSSI received at the cell edge $(10 \mathrm{~m})$ with TPmin is about $-56 \mathrm{dBm}$.
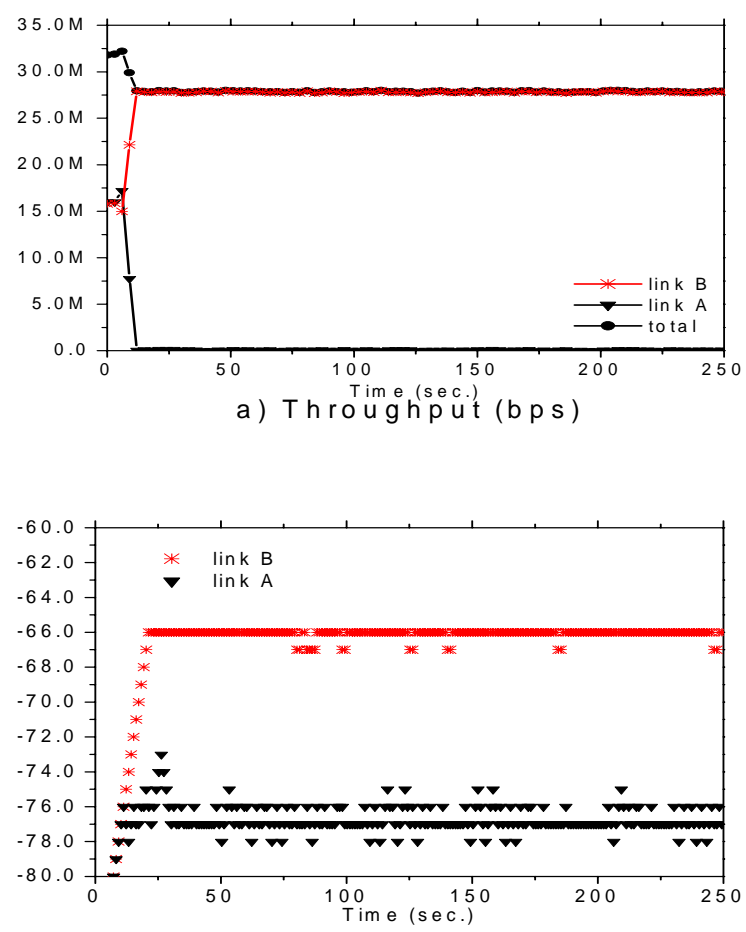

b) C C A threshold ( $\mathrm{dB}$ m)

Fig. 4 Link starvation with CCA adaptation only (54Mbps)

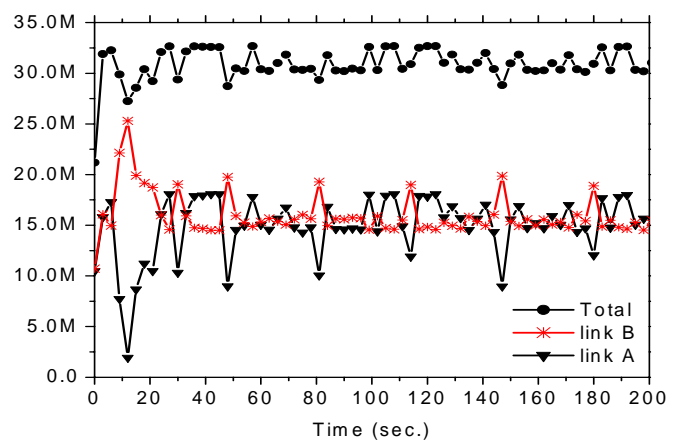

Fig.5 Regain fairness by TPC and CCA joint self-adaptation Fig. 4a shows the throughput of each link as well as the sum throughput when using CCA self-adaptation only. As expected, there is a severe fairness problem since the throughput of link A falls to almost zero. However, unlike what was reported in [8], the CCA threshold of link A as shown in Fig. 4b did not go to the minimum $(-86 \mathrm{dBm})-$ the main reason being that the unnecessary decrease of the CCA threshold has been effectively prevented by the proposed interference differentiation mechanism.

Fig. 5 shows the performance with adding TPC. Now two links are sharing the bandwidth equally. Fig. 6 traces how the CCA threshold and transmit power evolve with the adaptation. At the steady state, the transmit power of link A converges to a value $2 \mathrm{~dB}$ higher than that of link $\mathrm{B}$, sufficient to compensate the location difference between two links. On the other hand, the CCA threshold of link B drops to where it can 
sense link A's transmission. This figure also shows how Nth enters into exponential back-off to prevent oscillation.

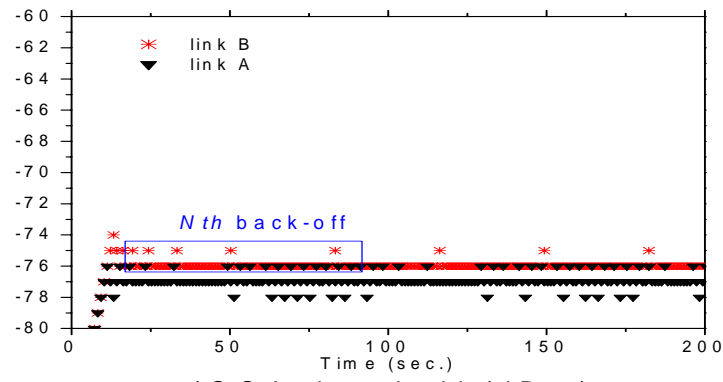

a) C C A threshold (dB m )

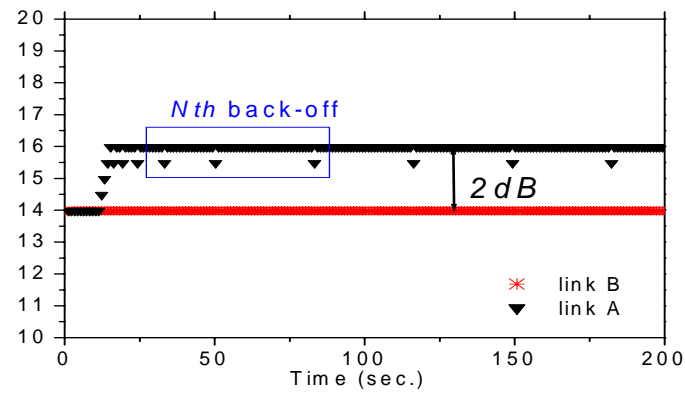

b) Transmission Power ( $\mathrm{dBm}$ )

Fig.6 Tracing CCA threshold and transmit power of the proposed TPC and CCA joint adaptation algorithm

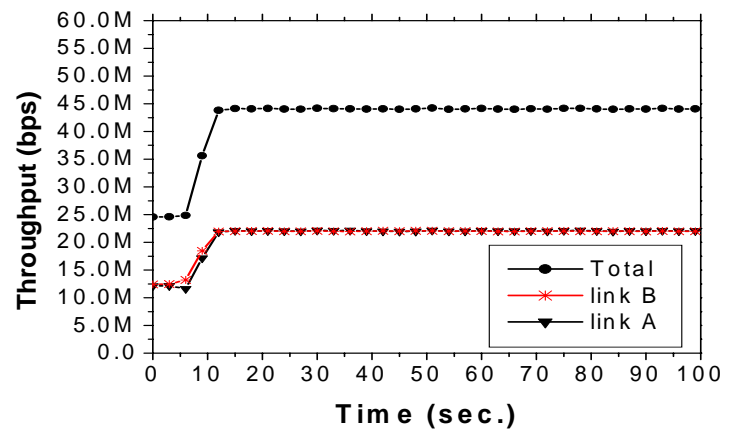

Fig.7 Improve throughput performance by CCA adaptation (36Mbps)

Last, Fig. 7 demonstrates how our algorithm leverages spatial reuse to improve network throughput. We change the rate from $54 \mathrm{Mbps}$ to $36 \mathrm{Mbps}$ so that TPC will not be triggered, because both links can now successfully transmit simultaneously due to additional link margin available at lower rate. As a result, total throughput almost doubles, from $25 \mathrm{Mbps}$ to $45 \mathrm{Mbps}$, with both links having equal share. In the future, we will further investigate the interaction between rate adaptation algorithms and our CCA/TPC solution.

\section{B. Multi-Cell Infrastructure WLAN}

Now let's look at a more realistic scenario and investigate the joint impact of the proposed TPC and CCA self-adaptation algorithms in a reasonably large-scale network. The topology comprises 10 cells with cell radius of 10 meters, AP-to-AP distance of 30 meters. Other parameters are the same as in the previous subsection. The resulting reuse distance (defined as the ratio of the AP-to-AP distance to the cell radius) is 3 , which is based on 3 orthogonal channels, e.g. $2.4 \mathrm{GHz}$ ISM band. Each cell has one AP-STA pair communicating; all are transmitting bi-directional saturated UDP traffic with packet size 2000 bytes (total of 20 uni-directional links). We use total throughput to measure system capacity, and worst link throughput to measure how link starvation has been avoided.

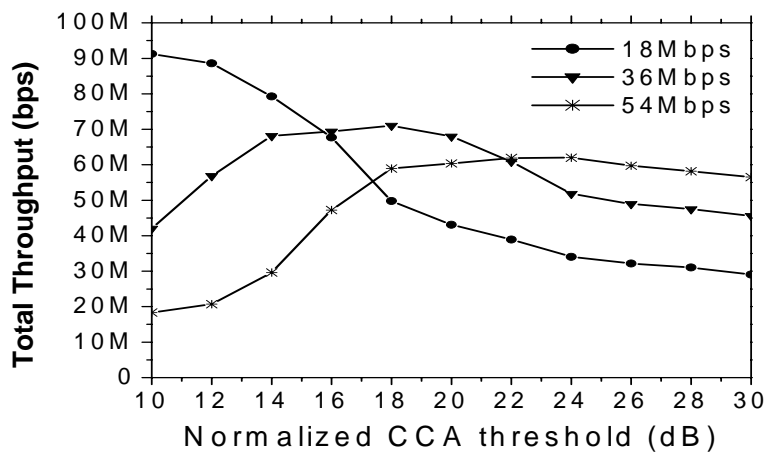

Fig.8 the optimal CCA threshold

Fig.8 shows the performance of the static setting, in which the same CCA threshold is used for all devices in the network. The $\mathrm{x}$-axis is CCA threshold (normalized by $\mathrm{RSSI}^{5}$, i.e., how much it is below RSSI) from $10 \mathrm{~dB}$ to $30 \mathrm{~dB}$, and the y-axis shows the total throughput.

Table1: Total Throughput

\begin{tabular}{|c|c|c|c|c|}
\hline Rate & Legacy & Ideal & CCA & CCA+TPC \\
\hline 54Mbps & 57Mbps & 62Mbps & $63 \mathrm{M}$ & 65M \\
\hline $36 \mathrm{Mbps}$ & $46 \mathrm{Mbps}$ & 71Mbps & $74 \mathrm{M}$ & 70M \\
\hline $18 \mathrm{Mbps}$ & 29Mbps & 91Mbps & $89 \mathrm{M}$ & 89M \\
\hline \multicolumn{5}{|c|}{ Table2: Worst Link Throughput } \\
\hline Rate & Legacy & Ideal & CCA & $\begin{array}{l}\text { CCA } \\
+ \text { TPC }\end{array}$ \\
\hline 54Mbps & 444Kbps & 1.3Mbps & 103Kbps & 752Kbps \\
\hline 36Mbps & 362Kbps & 1.2Mbps & $18 \mathrm{Kbps}$ & 586Kbps \\
\hline 18Mbps & $385 K b s$ & $182 \mathrm{Kbps}$ & 286Kbps & 646Kbps \\
\hline
\end{tabular}

For appropriate comparison, we use "Legacy" to denote no power control and no CCA adaptation, and all devices are using the minimum CCA threshold and the minimum transmit power ${ }^{6}$; "Ideal" means that all devices are using the same (minimum) transmit power and the same CCA threshold configured at the optimal value (obtained from Fig. 8). We use "CCA" to indicate the solution with the proposed CCA adaptation only, and "CCA+TPC" to indicate the solution with both CCA adaptation and TPC. Table 1 and 2 show the results of total throughput and worst link throughput, respectively. Clearly, both "CCA" and "CCA+TPC" achieve almost the same (> 95\%) performance as "Ideal" in terms of total throughput, much higher than "Legacy", e.g. 3x at 18Mbps. Furthermore, "CCA+TPC" dramatically improves worst link throughput compared to "CCA", e.g. 7x at 54Mbps. It is also better than "Legacy" for all three rates.

\footnotetext{
${ }^{5}$ at minimum transmit power.

${ }^{6}$ Here, "minimum transmit power" does not mean the hardware lower limit, for example $-1 \mathrm{dBm}$ in Cisco $1130 \mathrm{AP}$, but rather a default (starting) value that is below the maximum transmit power. It is $14 \mathrm{dBm}$ in our simulation.
} 
Fig. 9 shows the traces for CCA threshold and transmit power of the best link and the worst link at rate 36Mbps. In steady state, the worst link has CCA threshold about 2dB lower than the best link, while transmit power $1 \mathrm{~dB}$ higher. Two observations are made hereby: i) convergence of CCA threshold and transmit power is achievable with distributed self-adaptation; ii) the difference of CCA threshold or transmit power among links is small. In the future, we plan to conduct simulations with more scenarios.

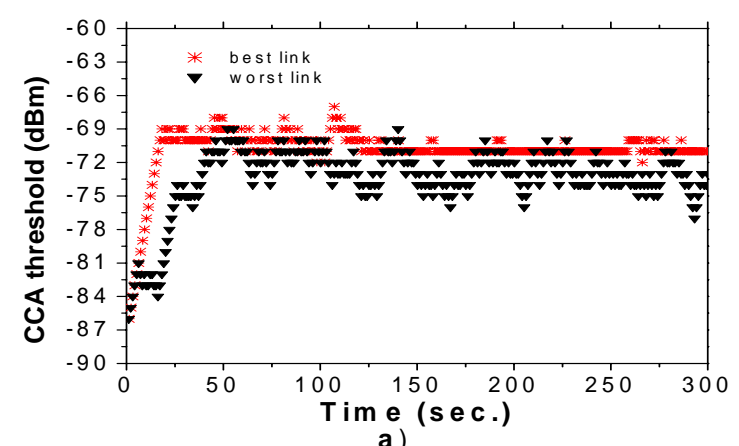

a)

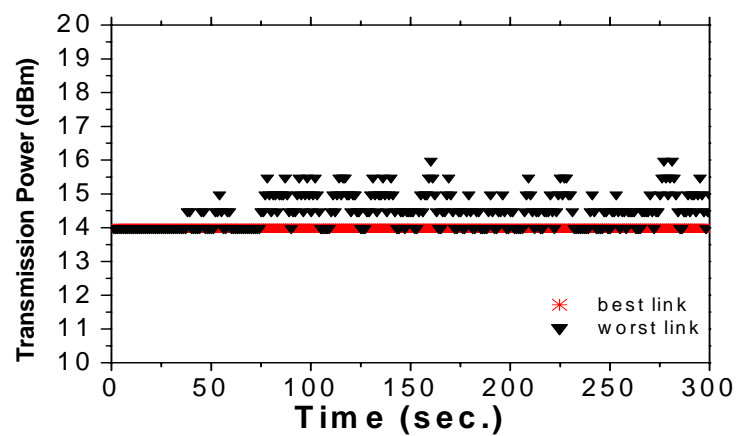

b)

Fig.9 tracing CCA threshold and transmit power

\section{RELATED WORK}

Recently, more and more research efforts [4] [12] have been dedicated to leveraging spatial reuse to improve network capacity of HD-WLAN. They fall into two major categories: CCA (clear channel assessment) adaptation and transmit power control (TPC). The former one tunes the energy level threshold that a transmitter uses to assess whether a channel is occupied, while the latter adjusts the actual power emitted in the air. We showed in [4] [6] by simulation and testbed experiment that network aggregate throughput can be dramatically improved by tuning the CCA threshold to an optimal value. Yang et al. [7] then proposed an analytical model to address the impact of MAC overhead on the optimal CCA threshold, considering both bandwidth independent overhead coming from PHY header, slot time, etc., and bandwidth dependent overhead caused mainly by collisions. Moreover, Zhan et al. [8] studied the impact of data rates, multi-hop forwarding, and other factors, such as SINR (signal to interference plus noise ratio), node topology, hidden/exposed terminal problems and bidirectional handshakes on determining the optimal carrier sensing range. The analytical approach of [9] provides the most concrete guidance for the optimal CCA threshold for single rate networks: setting the threshold such that the interference range equals the carrier sense range is a good, robust initialization.

TPC (transmit power control) approaches in 802.11 have also been well studied. In the adaptive power control scheme of [10], a station increases transmit power when successive packet losses are detected, and decreases it when the number of continuous successful transmissions exceeds a threshold. Muqattash et al. [11] uses an access window to allow for a series of RTS/CTS exchanges prior to data transmissions so that neighboring nodes can determine the optimal transmit power based on the information provided in the RTS and CTS packets to maximize spatial reuse.

To the best of our knowledge, there is very little work considering the joint impact of both transmit power and CCA threshold other than [12] which showed that the product of the transmit power and the CCA threshold should equal a constant for maximizing spatial reuse. However, how to prevent starvation of individual nodes while maximizing total throughput via self-adaptation was left unaddressed, which is the main contribution of this paper.

\section{CONCLUSIONS}

In this paper, we designed a practical solution for CCA adaptation and TPC under the constraint of no feedback control. Simple yet effective mechanisms were proposed for interference differentiation. Simulation with the two-cell scenario was used to comprehensively verify that our design can effectively address the hidden and exposed terminal problems. With the more realistic multi-cell scenario, we further evaluated the performance of the proposed solution. It was shown that our solution can achieve the same level of improvement in terms of total throughput as the ideal, while maintain higher worst link throughput than the legacy.

\section{REFERENCES}

[1] http://www.testmy.net/articles/article-89

[2] IEEE/ANSI. Wireless LAN MAC and PHY Layer Specifications, IEEE Std 802-11-1999, 1999.

[3]The Wireless Grid, Whitepaper, Aruba Wireless Networks, http://www.arubanetworks.com

[4] J. Zhu, X. Guo, L. L. Yang, W. S. Conner, and S. Roy and M. M. Hazra, Adapting physical carrier sensing to maximize spatial reuse in 802.11 mesh networks, Wirel. Commun. Mob. Comput. 2004, vol. 4, pp. 933-946.

[5] J. Zhu, X. Guo, L. Lily Yang, W. Steven Conner, Leveraging spatial reuse in 802.11 mesh networks with enhanced physical carrier sensing, ICC 2004.

[6] J. Zhu, B. Metzler, Y. Liu, and X. Guo, "Adaptive CSMA for Scalable Network Capacity in High-Density WLAN”, IEEE INFOCOM’06.

[7] X. Yang and N. H. Vaidya, "On the Physical Carrier Sense in Wireless Ad-hoc Networks", in Proc. IEEE INFOCOM 2005.

[8] H. Zhai and Y. Fang, Physical Carrier Sensing and Spatial Reuse in Multirate and Multihop Wireless Ad Hoc Networks, IEEE INFOCOM’06.

[9] S. Roy, H. Ma, R. Vijaykumar and J. Zhu, “'Optimizing 802.11 Wireless Mesh Network Performance Using Physical Carrier Sensing," UWashington Tech. Report, 2006.

[10] J. Chen et. al., PASA: Power Adaptation for Starvation Avoidance to Deliver Wireless Multimedia, IEEE JSAC, vol. 21, no. 10, Dec. 2003.

[11] A. Muqattash and M. Krunz, POWMAC: A Single-Channel PowerControl Protocol for Throughput Enhancement in Wireless Ad Hoc Networks., IEEE JSAC, vol. 23, no. 5, May 2005. 
[12] J. A. Fuemmeler, N. H. Vaidya, and V. V. Veeravalli, "Selecting Transmit Powers and Carrier Sense Thresholds for CSMA Protocols", UIUC Technical Report, Oct. 2004. 